\section{Diabetes mellitus induzido por quetiapina: relato de caso}

New onset diabetes associated with quetiapine treatment: case report

Sr. Editor,

Os antipsicóticos atípicos - especialmente a clozapina e a olanzapina - têm sido relacionados a distúrbios metabólicos como diabetes. ${ }^{1}$ Segue relato de paciente com diagnóstico de esquizofrenia, que desenvolveu quadro de diabetes mellitus após introdução da quetiapina.

ABC, 25 anos, masculino, iniciou quadro psicótico em maio de 2003, com idéias de caráter persecutório, autoreferência, alucinações auditivas e prejuízo no pragmatismo. Fez tratamento irregular com clorpromazina por quatro meses até fevereiro de 2004. Iniciou o uso de quetiapina 100 mg/dia em março de 2004; tem glicemia de jejum prévia ao início da quetiapina de $100 \mathrm{mg} / \mathrm{dl}$ (em 10/03/2004) e IMC de 39,19. A dose de quetiapina variou entre 100 e $600 \mathrm{mg} /$ dia; a partir de 18/05/04, foi introduzido haloperidol $5 \mathrm{mg} /$ dia e biperideno $2 \mathrm{mg} /$ dia devido à resposta parcial da quetiapina. Em 31/05/2004, o paciente deu entrada no pronto socorro com hipótese de cetoacidose diabética e diabetes mellitus. Na ocasião, apresentava náuseas, vômitos, dextro de 546, glicosúria, cetonúria e ph sanguíneo de 7,06. Paciente sem história prévia de diabetes e sem antecedentes familiares. A quetiapina foi suspensa e introduziram insulinoterapia, com melhora dos níveis glicêmicos; foram afastados quadros infecciosos. Teve alta em 07/06/2004, em uso de insulina NPH $110 \mathrm{UI} /$ dia em três doses, sendo encaminhado para a endocrinologia. Desde setembro/2004 em uso de metformina $850 \mathrm{mg}$ de 12/12 horas, insulina NPH $60 \mathrm{UI} / \mathrm{dia}$ em duas doses, insulina regular $6 \mathrm{UI} / \mathrm{dia}$, haloperidol $10 \mathrm{mg} / \mathrm{dia}$, biperideno $2 \mathrm{mg} / \mathrm{dia}$, clonazepam 2 mg/dia e sertralina 50 mg/dia, com IMC de 36,54 e glicemia de jejum de 333 mg/dl (em 29/09/04).

No caso da quetiapina, o primeiro relato de caso é de 1999. ${ }^{2}$ O paciente em questão apresentava diagnóstico de transtorno bipolar e, após início de quetiapina 200 mg/dia associada a outras medicações, desenvolveu quadro de diabetes, inclusive necessitando de insulina por cinco meses, quando houve remissão do quadro. Em levantamento realizado em 2002 com 38.632 pacientes com esquizofrenia 15.984 em uso de antipsicóticos convencionais e 22.648 em uso de antipsicóticos atípicos, sendo que 955 (4,2\%) estavam em uso de quetiapina -, encontrou uma freqüência de diabetes $9 \%$ maior nos pacientes em uso de antipsicóticos atípicos, especialmente naqueles que receberam clozapina, olanzapina e quetiapina. ${ }^{3}$ Entretanto, em estudo retrospectivo de 590 pacientes recebendo antipsicóticos típicos e atípicos, não encontrou aumento de glicemia nos pacientes em uso de quetiapina $(n=13) .^{4}$

Apesar de haver apenas relatos de casos mostrando a associação de quetiapina e diabetes, nossa recomendação seria evitar essa medicação em pacientes de maior risco para desenvolver alterações metabólicas, especialmente em pacientes obesos, devido ao risco de desenvolver a síndrome meta- bólica: obesidade abdominal, dislipidemia, hiperinsulinemia, hipertensão e hipercoagulabilidade. Além disso, é importante monitorar os níveis glicêmicos e lipídicos desses pacientes, com medidas antes do início da droga e em intervalos regulares nos primeiros seis meses, que é o período em que ocorrem com maior freqüência os eventos hiperglicêmicos.

Cecília Attux

Departamento de Psiquiatria, Universidade Federal de São Paulo (UNIFESP), São Paulo (SP), Brasil

Ana Cristina Chaves

Departamento de Psiquiatria e Programa de Primeiro Episódio Psicótico, Universidade Federal de São Paulo (UNIFESP), São Paulo (SP), Brasil

Financiamento: Inexistente

Conflito de interesses: Inexistente

\section{Referências}

1. de Sena EP, Sampaio AS, Quarantini L de C, Oliveira IR de. Diabetes mellitus and atypical antipsychotics. Rev Bras Psiquiatr. 2003;25(4):253-7

2. Sobel M, Jaggers ED, Franz MA. New-onset diabetes mellitus associated with the initiation of quetiapine treatment. J Clin Psychiatry. 1999;60(8):556-7.

3. Sernyak MJ, Leslie DL, Alarcon RD, Losonczy MF, Rosenheck R. Association of diabetes mellitus with use of atypical neuroleptics in the treatment of schizophrenia. Am J Psychiatry. 2002;159(4):561-6.

4. Wirshing DA, Boyd JA, Meng LR, Ballon JS, Marder SR, Wirshing WC. The effects of novel antipsychotics on glucose and lipid levels. J Clin Psychiatry. 2002;63(10):856-65.

\section{Osteoporose em pacientes esquizofrênicos: um problema negligenciado?}

Osteoporosis in schizophrenic patients a neglected issue?

\section{Sr. Editor,}

A osteoporose afeta cerca de $30 \%$ das mulheres no período pós-menopausa, sendo reconhecida como um problema de saúde pública. ${ }^{1}$ Uma vez que sua prevalência aumenta com a expansão da longevidade na população, é possível que no Brasil tenhamos uma maior incidência desta patologia nos próximos anos. Diversos fatores genéticos, ambientais e socioculturais aumentam o risco da osteoporose. Em pacientes esquizofrênicos, o uso prolongado de antipsicóticos, tanto típicos quanto atípicos, está sendo apontado como fator de risco adicional para osteoporose ou de diminuição da densidade mineral óssea (DMO). ${ }^{2-5}$ Estas drogas, em uso prolongado, foram demonstradas induzindo o aumento de prolactina a 